\title{
An investigation into the nutritional efficacy, tolerance and patient acceptance of pre-thickened nutritional supplements in dysphagic patients
}

\author{
M. Macleod ${ }^{1}$, L. Blackie ${ }^{2}$ and S. Humphrey ${ }^{3}$ \\ ${ }^{1}$ Learning Disabilities Team, Edinburgh, EH9 2AR, UK, ${ }^{2}$ West Lothian Community Learning Disabilities Team, \\ Edinburgh EH54 5EH, UK and ${ }^{3}$ Fresenius Kabi Ltd, Cheshire WA7 INT, UK
}

The fluid, energy and protein intakes of dysphagic patients on texture-modified diets have been shown to be inadequate ${ }^{(1-3)}$. The current treatment of malnourished, dysphagic patients is the use of texture modified diets, thickened fluids, and the prescription of oral nutritional supplements (ONS). Enteral tube feeding may have to be considered where it is not possible to meet nutritional requirements orally. It is necessary to manually thicken ONS to reduce the risk of aspiration. However, it is difficult to successfully thicken ONS to the recommended consistency with proprietary powder thickeners ${ }^{(4)}$. The purpose of this research was to investigate the nutritional efficacy, tolerance and patient acceptance of a new pre-thickened ONS in patients with dysphagia.

A 4-week open, non-controlled, prospective observational study was conducted with 24 subjects with learning disabilities. Subjects were identified by a speech and language therapist as requiring a modified textured diet with thickened fluids and by the dietitian as not meeting their nutritional requirements. Subjects were stratified into two groups according to their swallow ability and were assigned to either the pre-thickened supplement stage 1 (syrup) or stage 2 (custard) group ${ }^{(5)}$. Nutritional efficacy was measured by change in weight. Subjects were grouped by the dietitian according to whether the aim of treatment was to stabilise weight or gain weight. Tolerance was measured by change in gastrointestinal symptoms. Acceptability was assessed using a visual scale at the end of the 28 -day period. A subjective evaluation was completed for each patient after 28 days by the investigators and carers regarding the success of the prethickened supplement.

The results show a marginal increase in weight over the 28-day period. Weight gain in each group is shown in the Table below:

\begin{tabular}{lcc}
\hline Aim of treatment & Number of patients & Median weight gain $(\mathrm{kg})$ \\
\hline Stabilise weight & 12 & 0.15 \\
Gain weight & 8 & 0.40 \\
\hline
\end{tabular}

There was no evidence of a difference in gastrointestinal status over time. There was also no evidence of a difference regarding change in bowel frequency or change in stool type. Using a visual scale subjects scored the pre-thickened ONS highly for mouthfeel, smell, visual appearance and taste. The pre-thickened ONS was successful in 21/24 (87.5\%) subjects. The investigators outlined the key advantages of the pre-thickened ONS over existing treatment options as: ease of use (71\%), guaranteed and safer consistency $(71 \%)$, meets nutritional requirements $(58 \%)$ and compliance $(50 \%)$. Carers reported that the subjects found the drinks much easier to take and palatable.

Pre-thickened ONS may offer considerable advantages over current strategies in the nutritional support of patients with dysphagia. Therefore this approach can provide a potentially safer solution, meet nutritional requirements and offer more choice compared to the modification of existing oral nutritional supplements.

1. Vivanti AP (2009) J Hum Nutr Dietet 22, 148-155.

2. Wright L, Cotter D, Hickson M et al. (2005) J Hum Nutr Dietet 18, 213-219.

3. Whelan K (2001) Clin Nutr 20, 423-428.

4. Bedson JV (2009) Complete Nutr 9, 47-49.

5. British Dietetic Association and Royal College of Speech and Language Therapists (2002) National Descriptors for Texture Modification in Adults. Birmingham: British Dietetic Association. 